\title{
RESEARCH IN CONTROLLED MOVES OF ROBOTIC MANIPULATORS FOR BIOLOGICAL OBJECT PROCESSING
}

\author{
Daiva Nemeiksyte, Vitalijs Osadcuks \\ Latvia University of Life Sciences and Technologies, Latvia \\ nemeiksyte.daiva@gmail.com, vtl@tvnet.lv
}

\begin{abstract}
Modern robots are complex technical devices with the most valuable properties being the ability to perform certain tasks better, quicker and more accurately than a human being. Therefore, even there are many areas of robot application, they were first introduced in the areas requiring a lot of mechanical and simple manual work: milking robots; sheep shearing robots; maize field robots that cut pest-damaged leaves to prevent further spreading; mechanical weeding and micro-spraying robots; greening, seedbed preparation, spraying, growing are performed with smaller agricultural robots that navigate using GPS guidelines; handling robots; biological object detection robots. To be more specific, biological object detection robots in agriculture face numerous challenges as only few of the listed applications do not require robot movement, because in agriculture the majority of operations are related to movement of machines. Moreover, in contrast to industry, when performing the assigned tasks they need to interfere with various plants, soil, irregularities of various types, biological objects, etc., which impairs their accuracy and mobility and results in different power required for the same action. Therefore, the subject of the study in this article is a robotic arm for detecting and removing/eliminating biological objects, and the goal of the study was to develop the methods for managing the movement-dynamics system as well as for planning the trajectory of the robotic arm ensuring the manipulator will detect biological objects retaining wide enough movement spectrum and consistency of movement modes under the influence of external uncontrolled disturbances. Based on the above, a model of a robotic arm was developed after calculating the missing parameters for the engines of the experimental robotic arm, which was able to generate a control signal and calculate the linear and angular velocities and accelerations of the kinematic chain motion characteristics, linear accelerations of the chain centres of mass, and the coordinates of the robotic arm tip. The developed model allows optimization of the manipulator's operation and supports constant trajectory of the robot working tool (camera and laser).
\end{abstract}

Keywords: manipulator dynamics, $6 \mathrm{DOF}$, direct position task, planning the trajectory, mathematical modelling.

\section{Introduction}

Currently, there are two dominant directions of robot design in robotics. One of them concerns designing universal multifunctional robots which carry out more technological operations, have more degrees of freedom [1;2]; however, it is much more difficult to achieve higher accuracy of their positioning and reliability of work, and they are more expensive to manufacture. Another direction of robot design, namely, designing of specialized and special-purpose robots, will be discussed in this article $[3 ; 4]$. Such robots perform certain technological operations, carry out servicing of specific devices; they have less degrees of freedom, are accurate, fast, and reliable.

The purpose of the robotic arm discussed in the article is to demonstrate the scope of capabilities of the modern robotics. At the same time, it is aimed to break down the stereotype in society that the capabilities of a robotic arm are limited to lifting, pushing or otherwise manipulate certain objects in a primitive way. The robotic arm being discussed is intended to detect biological objects with the help of a camera and a laser having the purpose to eliminate them.

\section{Materials and methods}

The system being designed consists of a robotic arm with seven degrees of freedom with the block diagram presented in Fig. 1a. The position of the end point (laser with a camera) of the robotic arm is altered by adjusting angular velocities of the six (M1...M2) DC motors. Motor seven (M7) adjusts the position of the robotic arm in the operating zone. For measuring the joint rotation angle incremental optical converters (S1...S7) were used. All these components are connected to the control unit - the micro-controller, which receives control commands and data from the PC.

The structure of the system being designed shall consist of the following (Fig. $1 \mathrm{~b}$ ).

- UR10 Robotic manipulator (Universal Robots), which has 6 degrees of freedom and tree flexible elbow joints; these joints are capable of rotating 360 degrees without stopping. The length of fully extended arm is approx. 1.3 metres. UR10 robotic arm can be programmed 
using special branded tablet, or manually. The robot is moved to the required positions, which are then memorized and repeated independently. If the robotic arm detects external resistance of $5 \mathrm{~kg}$ or more, it stops working immediately. This means the robot can be easily stopped with one finger.

- The end point devices of the robotic arm include a laser with a camera. The camera is used for detecting and determining biological objects, and the laser is used for eliminating them.

- The manipulator is mounted on a base, which by rotating the toothed gear will allow the robot moving horizontally along the rail frame on a $1.5 \mathrm{~m}$ high platform.

- Robotic arm can be controlled on two levels: manual operation using a remote control; and autonomous mode with program instructions executed sequentially; as well as self-functioning hardware system for protection against breaking.

a)

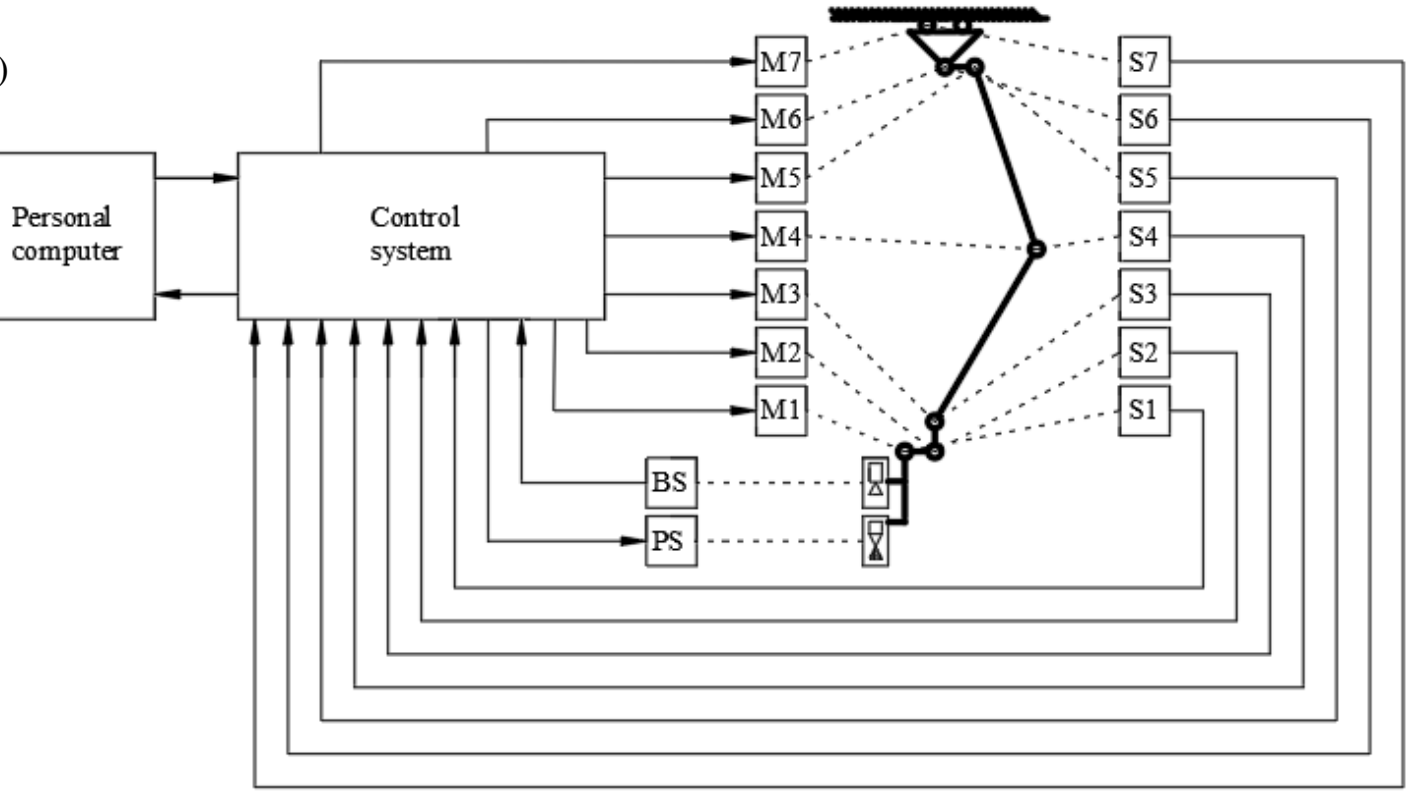

b)

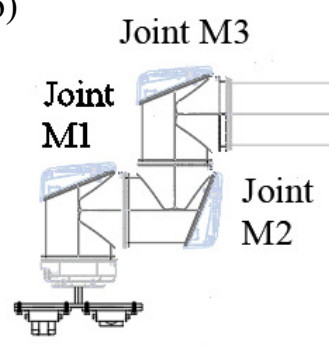

Camera Laser

BS PS

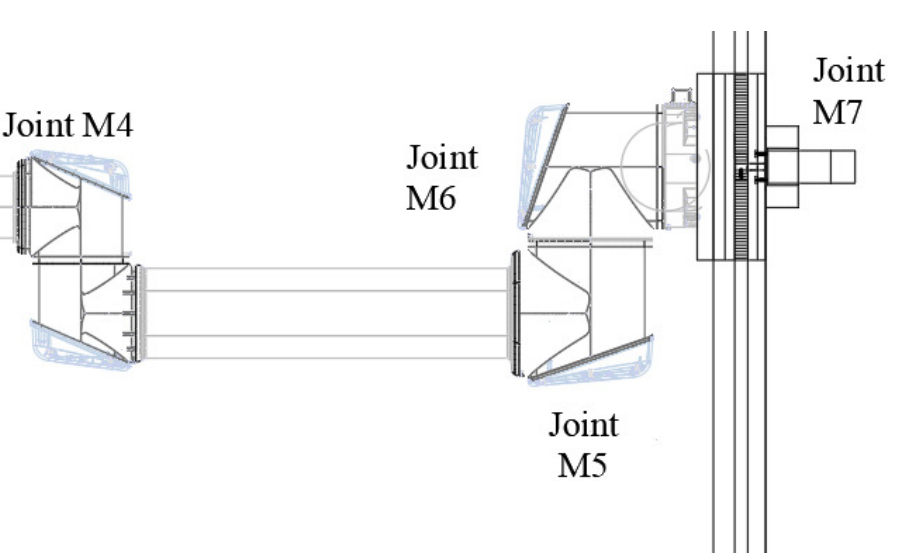

Fig. 1. Robotic arm with 6 degrees of freedom: a - block diagram; b - structure diagram

\section{Mathematical model of the robotic arm}

While designing a robot's single- or multi-joint control system, it is essential to analyse the robot's dynamics with the help of equations of movement. These equations are used for simulating the robot's movement, movement law selection and assessment of the quality of the kinematic diagram. Therefore, it is necessary to compose suitable equations of movement for operating the robot of the system being designed. The dynamics of the robotic arm being designed and discussed can be described by the following system of equations $[5 ; 6]$ : 


$$
\left\{\begin{array}{l}
J_{2} \ddot{q}_{2}+m_{2} l_{1} r_{2 c} \ddot{q}_{1} \cos \left(q_{2}-q_{1}\right)-m_{2} l_{1} r_{2 c} \dot{q}_{1}\left(\dot{q}_{2}-\dot{q}_{1}\right) \sin \left(q_{2}-q_{1}\right)=T_{1} ; \\
\left(J_{1}+m_{2} l_{1}^{2}\right) \ddot{q}_{1}+m_{2} l_{1} r_{2 c} \ddot{q}_{2} \cos \left(q_{2}-q_{1}\right)-m_{2} l_{1} r_{2 c} \dot{q}_{2}\left(\dot{q}_{2}-\dot{q}_{1}\right) \sin \left(q_{2}-q_{1}\right)=T_{2} ; \\
\left(J_{2}+m_{3} l_{2}^{2}\right) \ddot{q}_{2}+m_{3} l_{2} r_{3 c} \ddot{q}_{3} \cos \left(q_{3}-q_{2}\right)-m_{3} l_{2} r_{3 c} \dot{q}_{3}\left(\dot{q}_{3}-\dot{q}_{2}\right) \sin \left(q_{3}-q_{2}\right)=T_{3} ; \\
\left(J_{3}+m_{4} l_{3}^{2}\right) \ddot{q}_{3}+m_{4} l_{3} r_{4 c} \ddot{q}_{4} \cos \left(q_{4}-q_{3}\right)-m_{4} l_{3} r_{4 c} \dot{q}_{4}\left(\dot{q}_{4}-\dot{q}_{3}\right) \sin \left(q_{4}-q_{3}\right)=T_{4} ; \\
\left(J_{4}+m_{5} l_{4}^{2}\right) \ddot{q}_{4}+m_{5} l_{4} r_{5 c} \ddot{q}_{5} \cos \left(q_{5}-q_{4}\right)-m_{5} l_{4} r_{5 c} \dot{q}_{5}\left(\dot{q}_{5}-\dot{q}_{4}\right) \sin \left(q_{5}-q_{4}\right)=T_{5} ; \\
\left(J_{5}+m_{6} l_{5}^{2}\right) \ddot{q}_{5}+m_{6} l_{5} r_{6 c} \ddot{q}_{6} \cos \left(q_{6}-q_{5}\right)-m_{6} l_{5} r_{6 c} \dot{q}_{6}\left(\dot{q}_{6}-\dot{q}_{5}\right) \sin \left(q_{6}-q_{5}\right)=T_{6} ;
\end{array}\right.
$$

where $J_{1}=l_{1}^{2} m_{1} / 3, J_{2}=l_{2}{ }^{2} m_{2} / 3, J_{3}=l_{3}^{2} m_{3} / 3, J_{4}=l_{4}{ }^{2} m_{4} / 3, J_{5}=l_{5}{ }^{2} m_{5} / 3, J_{6}=l_{6}{ }^{2} m_{6} / 3-$ the values of joint inertia momentum, when the joints are rotating around their axes of rotation, $\mathrm{kg} \cdot \mathrm{m}^{2}$; $r_{1 c}=l_{1} / 2, r_{2 c}=l_{2} / 2, r_{3 c}=l_{3} / 2, r_{4 c}=l_{4} / 2, r_{5 c}=l_{5} / 2, r_{6 c}=l_{6} / 2-$ the distance from the joint's center of mass, m (see Fig. 2);

$m_{1,2,3,4,5,6}$ - masses of joints, $\mathrm{kg}$;

$l_{1,2,3,4,5,6}$ - lengths of joints, $\mathrm{m}$;

$q_{1,2,3,4,5,6}$ - joint rotation angle, rad;

$\dot{q}_{1,2,3,4,5,6}$ - joint angular velocity, $\mathrm{rad} \cdot \mathrm{s}^{-1}$;

$\ddot{q}_{1,2,3,4,5,6}$ - joint angular acceleration, $\mathrm{rad} \cdot \mathrm{s}^{-2}$;

$\mathrm{g}-$ free fall acceleration, $\mathrm{m} \cdot \mathrm{s}^{-2}$;

$T_{1,2,3,4,5,6}$ - torques at the axes of joint connections, $\mathrm{Nm}$.

The position of the end point of the robotic arm is altered by adjusting the motors M1...M6, which generate the torques and thus rotate separate links of the robotic arm around their axes. DC motor has its own electrical and electro-mechanical time constants. Their impact on the arm movement can be assessed by complementing the mathematical model of the arm with the mathematical model of the DC motor. The forces of static friction and fluid friction affecting the movement during the reverse and while moving at maximum speeds need to be assessed. Mathematical model of the DC motor taking into account the forces of friction $[7 ; 8]$ :

$$
\left\{\begin{array}{l}
L \frac{\mathrm{d} i}{\mathrm{~d} t}=u-i r-\omega C_{E} \\
J \frac{\mathrm{d} \omega}{\mathrm{d} t}=i C_{M}-M_{A}-\omega f_{S}-\left[T_{C} \operatorname{sign}(\omega)+\left(T_{S}-T_{C}\right) \exp (-\alpha|\omega|) \operatorname{sign}(\omega)\right]
\end{array}\right.
$$

where $L$-inductance of the motor's anchor chain, $\mathrm{H}$;

$i$ - strength of electric current flowing through the anchor chain A;

$u$ - voltage connected to the anchor chain, V;

$r$ - resistance of the anchor chain, $\Omega$;

$\omega-$ angular rotation speed of the motor shaft, $\mathrm{rad} \cdot \mathrm{s}^{-1}$;

$C_{E}-$ generic electrical constant, $\mathrm{V} \cdot \mathrm{s} \cdot \mathrm{rad}^{-1}$;

$J$ - moment of inertia of the motors; anchor, $\mathrm{kg} \cdot \mathrm{m}^{2}$;

$C_{M}$ - electromechanical transmission coefficient, $\mathrm{Nm} \cdot \mathrm{A}^{-1}$;

$M_{A}$ - moment of resistance, $\mathrm{Nm}$;

$f_{S}-$ fluid friction coefficient, $\mathrm{Nm} \cdot \mathrm{s} \cdot \mathrm{rad}^{-1}$;

$T_{C}-$ moment of dry fiction, $\mathrm{Nm}$;

$T_{S}-$ moment of static fiction, $\mathrm{Nm}$;

$\alpha$ - friction constant, s.

The joints of the robotic arm only perform rotational movements. However, the final movement of the robotic tool is linear. The $\mathrm{x}$ and $\mathrm{y}$ position of the end point of the robot arm on the Cartesian coordinate system is calculated as follows $[9 ; 10]$ : 


$$
\left\{\begin{array}{l}
x=l_{1} \cos \left(q_{1}\right)+l_{2} \cos \left(q_{1}+q_{2}\right)+l_{3} \cos \left(q_{1}+q_{2}+q_{3}\right)+l_{4} \cos \left(q_{1}+q_{2}+q_{3}+q_{4}\right)+ \\
+l_{5} \cos \left(q_{1}+q_{2}+q_{3}+q_{4}+q_{5}\right)+l_{6} \cos \left(q_{1}+q_{2}+q_{3}+q_{4}+q_{5}+q_{6}\right) ; \\
y=l_{1} \sin \left(q_{1}\right)+l_{2} \sin \left(q_{1}+q_{2}\right)+l_{3} \sin \left(q_{1}+q_{2}+q_{3}\right)+l_{4} \sin \left(q_{1}+q_{2}+q_{3}+q_{4}\right) \\
+l_{5} \sin \left(q_{1}+q_{2}+q_{3}+q_{4}+q_{5}\right)+l_{6} \sin \left(q_{1}+q_{2}+q_{3}+q_{4}+q_{5}+q_{6}\right) .
\end{array}\right.
$$

Generalized dynamic model of a robotic arm of 6 degrees of freedom is shown in Figure 2. Model developed in Matlab Simulink environment consists of several units:

- units "Motor1", "Motor2", "Motor3", "Motor4", "Motor5", "Motor6" are the models of the DC motor developed according to the formula (1);

- unit "Arm dynamics" is construed according to the formula (2);

- unit "Direct positioning task" is construed according to the formula (3).

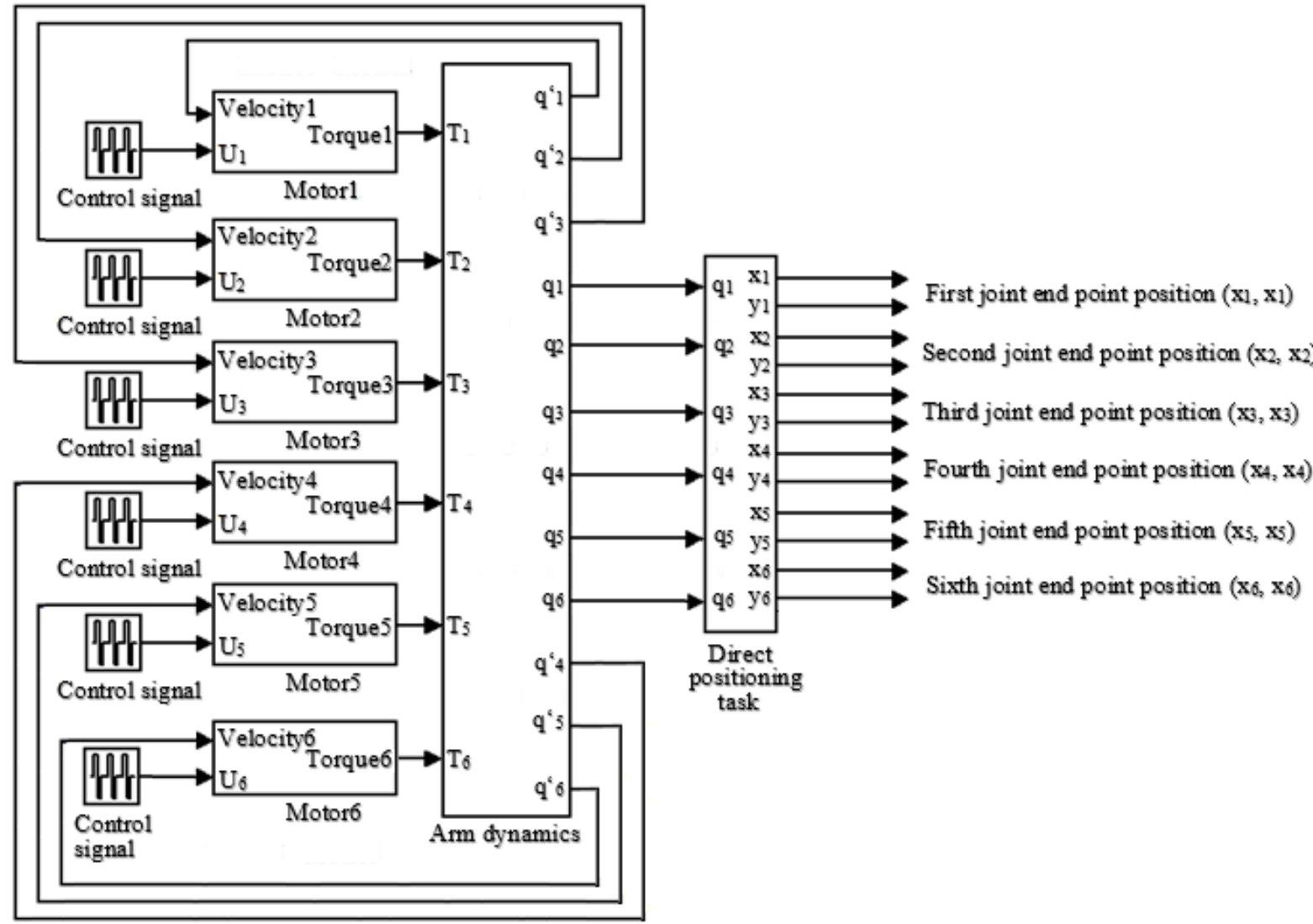

Fig. 2. Model of robotic arm with six degrees of freedom

\section{Results and discussion}

For designing a dynamic model of a robotic arm, the lengths and masses of the joints are also required: $l_{1}=0.0342 \mathrm{~m} ; \quad m_{1}=7.1 \mathrm{~kg} ; \quad l_{2}=0.411 \mathrm{~m} ; \quad m_{2}=12.7 \mathrm{~kg} ; \quad L_{3}=0.249 \mathrm{~m} ; \quad m_{3}=4.27 \mathrm{~kg}$; $l_{4}=0.019 \mathrm{~m} ; m_{4}=2 \mathrm{~kg} ; L_{5}=0.019 \mathrm{~m} ; m_{5}=2 \mathrm{~kg} ; l_{6}=0.026 \mathrm{~m} ; m_{2}=0.365 \mathrm{~kg}$.

The dynamics of each joint was examined separately. The motor of the sixth joint applied the rated voltage for one second. Then the polarity of the voltage is changed and the motor reverses - it changes its direction of rotation. After another second, the voltage applied to the sixth engine became zero and the engine was stopped. Such experiment allows observing the processes of acceleration, reversing and braking in a consistent way. During the simulation experiment the voltage of the M1, M2, M3, M4 and M5 joint motor was zero during the entire test. Simulation results are presented in Figure 3. 


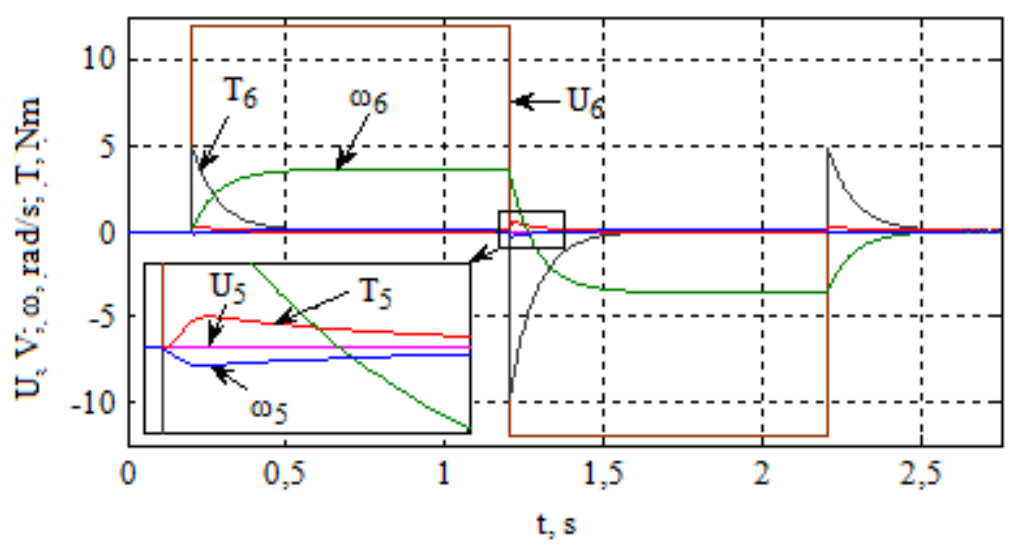

Fig. 3. Robotic arm simulation results: $U_{1}, U_{2}, U_{3}, U_{4}, U_{5}, U_{6}$ - variation of motor control voltage; $\omega_{1}, \omega_{2}, \omega_{3}, \omega_{4}, \omega_{5}, \omega_{6}-$ variation of motor angular velocity; $T_{1}, T_{2}, T_{3}, T_{4}, T_{5}, T_{6}$ - motor-generated torques

In the same way, the dynamics of the first joint was studied. During the simulation experiment the voltage of the M2, M3, M4, M5 and M6 joint motor was zero during the entire test. Simulation results are presented in Figure 4.

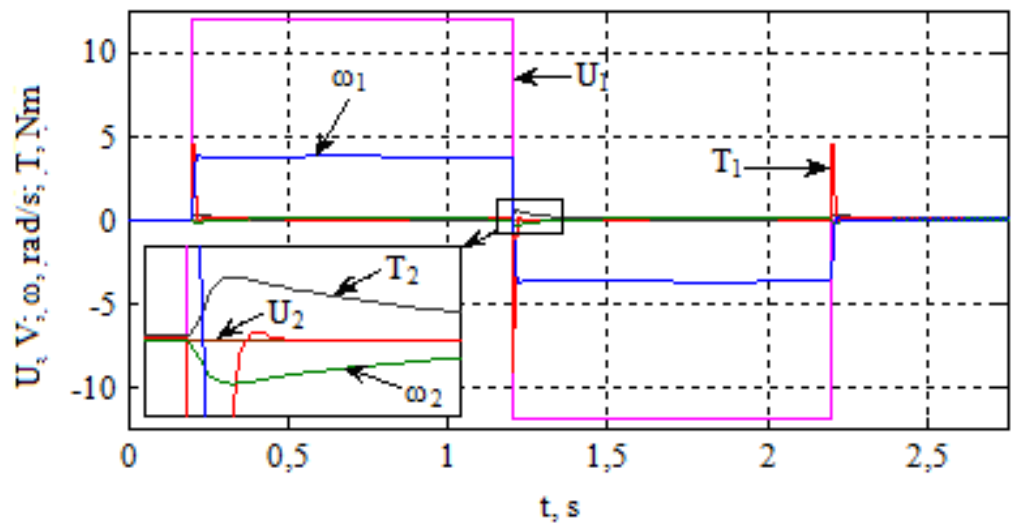

Fig. 4 Robotic arm simulation results: $U_{1}, U_{2}, U_{3}, U_{4}, U_{5}, U_{6}$ - variation of motor control voltage; $\omega_{1}, \omega_{2}, \omega_{3}, \omega_{4}, \omega_{5}, \omega_{6}$ - variation of motor angular velocity; $T_{1}, T_{2}, T_{3}, T_{4}, T_{5}, T_{6}$ - motor-generated torques

\section{Conclusions}

The modeling systems for robotic arms operating in a system of six degrees of freedom, which are superior to the robots of other design types, because they need less space and are characterized by better manoeuvrability, are in essence based on analytical and simulation modeling, and the operation can be implemented in a variety of ways using various technical components, taking into account the number of kinematic joints that make up the robot, the transmission mechanisms and their applicability. Embedded computers that make up the center of such robot control systems perform tasks faster and are more stable than a conventional computer, even if they have a narrow specialization.

When designing the system, a robotic arm UR10 with 6 degrees of freedom was selected with the elements mounted on its end point - camera and laser. The manipulator is mounted on a base, which by rotating the toothed gear will allow movement of the robot along the rail frame in the operating zone. All the above-mentioned components will be connected to the robotic arm control unit controller - which will control all the functions performed by the robot and commutate with the external PC.

After calculating the missing parameters of the robotic arm motors, a model of a robotic arm with 6 degrees of freedom was designed using the software package Matlab Simulink, which allows setting 
the control voltages and observing the variation of current, momentum, joint speed, and trajectory in the course of time.

\section{References}

[1] Lee K., Lee J., Woo B., Lee J., Lee Y. and Ra S. Modeling and Control of a Articulated Robot Arm with Embedded Joint Actuators. Proceedings of International conference "Information and Communication Technology Robotics (ICT-ROBOT)", September 6-8, 2018, Busan, South Korea, pp. 1-4.

[2] Li S., Wang Z., Zhang Q. and Han F. Solving Inverse Kinematics Model for 7-DoF Robot Arms Based on Space Vector. Proceedings of International conference "Control and Robots (ICCR)", September 15-17, 2018, Hong Kong, China, pp. 1-5.

[3] Xie Y., Pan J., Yang J. and Li J. Design of the fast speed two-arm robot in limited space. Proceedings of International conference "Robotics and Biomimetics (ROBIO)", December 5-8, 2017, Macau, China, pp. 652-656.

[4] Belda K. and Rovný O. Predictive control of 5 DOF robot arm of autonomous mobile robotic system motion control employing mathematical model of the robot arm dynamics. Proceedings of International conference "Process Control (PC)", June 6-9, 2017, Strbske Pleso, Slovakia, pp. 339-344.

[5] Parvathi S. and Selvi S. T. Design and fabrication of a 4 Degree of Freedom (DOF) robot arm for coconut harvesting. Proceedings of International conference "Intelligent Computing and Control (I2C2)", June 23-24, Coimbatore, India, pp. 1-5.

[6] Cao F., Liu J., Zhou C., Zhao Y., Fu Z. and Yan W. A Novel 5-DOF welding robot based on SCARA. Proceedings of International conference "Industrial Electronics and Applications (ICIEA)", June 15-17, 2015, Auckland, New Zealand, pp. 2016-2019.

[7] Herbin P. and Pajor M. Interactive 7-DOF motion controller of the operator arm (ExoArm 7DOF). Proceedings of International conference "INnovations in Intelligent SysTems and Applications (INISTA)", July 3-5, 2017, Gdynia, Poland, pp. 185-188.

[8] Tsai C., Deng P., Chan C. and Lin C. Fast position and posture control of an anthropomorphous 7 DOF dual-arm mobile robot. Proceedings of International conference "Automatic Control Conference (CACS)", December 2-4, 2013, Nantou, Taiwan, 2013, pp. 227-232.

[9] Kormushev P., Demiris Y. and Caldwell D. G. Kinematic-free position control of a 2-DOF planar robot arm. Proceedings of International conference "Intelligent Robots and Systems (IROS)" Sept. 28 - Oct. 2, 2015, Hamburg, Germany, 2015, pp. 5518-5525.

[10] Bouzgou K. and Ahmed-Foitih Z. Geometric modeling and singularity of 6 DOF Fanuc 200IC robot. Proceedings of International conference "Innovative Computing Technology (INTECH 2014)", 13-15 August 13-15, Luton, UK, pp. 208-214. 